\title{
Testing nonlinearity: Decision rules for selecting between logistic and exponential STAR models
}

\author{
Álvaro Escribano ${ }^{1}$, Oscar Jordá ${ }^{2}$ \\ 1 Department of Economics, Universidad Carlos III de Madrid, c/ Madrid 126, Madrid 28903, Spain \\ (e-mail: alvaroe@eco.uc3m.es) \\ 2 Department of Economics, University of California, One Shields Avenue, Davis, CA 95616-8578, \\ USA (e-mail: ojorda@ucdavis.edu)
}

\begin{abstract}
A new LM specification procedure to choose between Logistic and Exponential Smooth Transition Autoregressive (STAR) models is introduced. The new decision rule has better properties than those previously available in the literature when the model is ESTAR and similar properties when the model is LSTAR. A simple natural extension of the usual LM-test for linearity is introduced and evaluated in terms of power. Monte-Carlo simulations and empirical evidence are provided in support of our claims.
\end{abstract}

\section{JEL classification: $\mathrm{C} 12, \mathrm{C} 22$}

Key words: LM linearity tests, smooth transition autoregressive models, nonlinear models

\section{Introduction}

This paper introduces a simple decision rule for selecting between alternative Smooth Transition Autoregressive (STAR) models which improves the one suggested by Teräsvirta (1994). STAR models are a general class of state-dependent non-linear time-series models in which the transition between states is endogenously generated. ${ }^{1}$ Together with Hamilton's (1989) regime-switching model, where the transition between states is exogenously determined by a Markov

The first author acknowledges support from Spanish DGCIYT, PB98-0030 and TMR ERB-4061 PC97-0994. The second author acknowledges support from "La Caixa" and the Bank of Spain. We wish to thank two anonymous referees, R. F. Engle, J. Gonzalo, C. W. J. Granger, J. D. Hamilton and T. Teräsvirta for useful comments and suggestions.

${ }^{1}$ In addition, STAR models encompass other popular families of non-linear time-series models such as the Threshold Autoregressive (TAR) and the Exponential Autoregressive (EAR). See Haggan and Ozaki (1981), Tong (1983), Tsay (1989) and Granger and Teräsvirta(1993).

Corresponding author Á. Escribano 
Chain, state-dependent models are "reduced-form" models that allow for different dynamic responses that depend on the "state." Consequently, these models are particularly well suited to accommodate the asymmetric behavior of economic fluctuations recently documented in a variety of studies. ${ }^{2}$

By relying on a Taylor series approximation of the transition function (between states) around the scale parameter, ${ }^{3}$ we introduce a new specification strategy to choose between logistic and exponential STAR models. This alternative strategy is simpler and more successful in selecting the correct ESTAR model while avoiding the pitfalls (detailed below) of the procedure proposed in Teräsvirta (1994). Additional results concern the power of the nonlinearity tests discussed in Teräsvirta (1994) for which we offer some comments and alternatives. ${ }^{4}$ All the claims will be supported by Monte Carlo evidence and by a well known empirical example based on Teräsvirta and Anderson (1992).

The paper is organized as follows: Sect. 2 briefly reviews STAR models and nonlinearity testing. Section 3 discusses the decision rule proposed by Teräsvirta (1994) and introduces the alternative procedure suggested in this paper. Section 4 reports on the small sample properties of the alternative test procedures using Monte-Carlo techniques. Section 5 presents an empirical application, and Sect. 6 concludes.

\section{STAR models and nonlinearity testing}

\subsection{Overview}

Consider the following STAR model:

$$
y_{t}=\boldsymbol{\pi}^{\prime} \mathbf{x}_{t}+F\left(z_{t-d}, \gamma, c\right) \boldsymbol{\Theta}^{\prime} \mathbf{x}_{t}+u_{t}
$$

where $y_{t}$ is a scalar; $\mathbf{x}_{t}=\left(1, y_{t-1}, \ldots, y_{t-p}\right)^{\prime}=\left(1, \widetilde{\mathbf{x}}_{t}^{\prime}\right)^{\prime} ; \boldsymbol{\pi}^{\prime}=\left(\pi_{0}, \pi_{1}, \ldots, \pi_{p}\right)=$ $\left(\pi_{0}, \tilde{\boldsymbol{\pi}}^{\prime}\right) ; \boldsymbol{\Theta}^{\prime}=\left(\Theta_{0}, \Theta_{1}, \ldots, \Theta_{p}\right)=\left(\Theta_{0}, \widetilde{\boldsymbol{\Theta}}^{\prime}\right)$ and $1 \leqslant d \leqslant p . z_{t-d}$ is usually chosen to be $y_{t-d}$, although it could be any other predetermined or exogenous variable. $u_{t}$ is a martingale difference sequence with constant variance. ${ }^{5}$ It is common practice to assume that the process in (1) is stationary and ergodic, see Granger and Teräsvirta (1993) and Teräsvirta (1998). Gallant (1987) gives conditions to obtain the consistency and asymptotic normality of least mean distance estimators. Alternatively, Mira and Escribano (2000) give explicit conditions on the nonlinear function $\mathrm{F}$ (.) for $y_{t}$ to be near epoch dependent (NED) and also to obtain consistent and asymptotically normal nonlinear least squares parameter estimates.

\footnotetext{
${ }^{2}$ See Neftçi (1984), Rothman (1991), and Teräsvirta and Anderson (1992) for example.

${ }^{3}$ Luukkonen et al. (1988a), based on Davies (1977), introduced this solution for STAR models.

${ }^{4}$ Note: this alternative is an assumption imposed by the practitioner that permits narrowing down general nonlinearity into a workable family of non-linear models, namely STAR models.

5 This assumption is usually introduced to simplify the derivation of the asymptotic distribution of the LM test, see White (1984).
} 
In what follows we assume that the function $F\left(z_{t-d}, \gamma, c\right)$ is at least fourth order continuously differentiable with respect to the scale parameter $\gamma$. The exponential STAR model ${ }^{6}$ (ESTAR)has a transition function $F$, defined by

$$
F\left(z_{t-d}, \gamma, c\right)=\left[1-\exp \left\{-\gamma\left(z_{t-d}-c\right)^{2}\right\}\right] .
$$

The logistic STAR model ${ }^{7}$ (LSTAR) has as transition function:

$$
F\left(z_{t-d}, \gamma, c\right)=\left[\left\{1+\exp \left(-\gamma\left(z_{t-d}-c\right)\right)\right\}^{-1}-\frac{1}{2}\right] .
$$

Testing linearity against STAR-type nonlinearity implies testing the null hypothesis, $H_{0}: \boldsymbol{\Theta}^{\prime}=\mathbf{0}$ in (1). However, under the null, the parameters $\gamma$ and $c$ are not identified. Alternatively, we could choose $H_{0}^{*}: \gamma=0$ as our null hypothesis in which case neither $c$ nor $\boldsymbol{\Theta}^{\prime}$ would be identified. Davies (1977) first showed that conventional maximum likelihood theory is not directly applicable to this problem. A solution proposed in Luukkonen et al. (1988a) and adopted in Teräsvirta (1994) is to replace $F\left(z_{t-d}, \gamma, c\right)$ with a suitable Taylor series approximation. Under the null of linearity, the LM test is shown to possess the usual $\chi^{2}$ distribution asymptotically. ${ }^{8}$

In practice, the score test is performed by constructing the following auxiliary regression:

$$
y_{t}=\boldsymbol{\pi}^{\prime} \mathbf{x}_{t}+F_{\gamma}\left(z_{t-d}, \gamma=0, c\right) \boldsymbol{\Theta}^{\prime} \mathbf{x}_{t} \gamma+v_{1 t}
$$

where $F_{\gamma}($.$) indicates the first derivative of F\left(z_{t-d}, \gamma=0, c\right)$ with respect to $\gamma$. Substituting the expression for $F_{\gamma}($.$) into (4) gives:$

$$
y_{t}=\delta_{0}+\boldsymbol{\delta}_{1}^{\prime} \widetilde{\mathbf{x}}_{t}+\boldsymbol{\beta}_{1}^{\prime} \widetilde{\mathbf{x}}_{t} z_{t-d}+\boldsymbol{\beta}_{2}^{\prime} \widetilde{\mathbf{x}}_{t} z_{t-d}^{2}+v_{1 t}
$$

where the null hypothesis of linearity becomes $H_{0}^{\prime}: \boldsymbol{\beta}_{1}^{\prime}=\boldsymbol{\beta}_{2}^{\prime}=\mathbf{0}$. Call this test NL2. In the special case where $F($.$) is the logistic function \boldsymbol{\beta}_{2}^{\prime}=\mathbf{0}$. The process in (5) can be explosive and generally it is not a meaningful time series model ${ }^{9}$ (see Granger and Andersen 1978). Luukkonen et al. (1988a) realized that this test could have low power against alternatives where $\widetilde{\Theta}^{\prime}$ is "small" and $\Theta_{0}$ is "large" in absolute value if the model is LSTAR. Consider a case where the nonlinearity is active due to the parameter $\Theta_{0}$ exclusively. In this case since the parameter vector $\boldsymbol{\beta}_{1}^{\prime}$ is independent of $\Theta_{0}$ the NL2 test has no power. To overcome this difficulty, they proposed to include up to third order powers in the auxiliary regression since for the LSTAR model the parameter vector $\boldsymbol{\beta}_{3}^{\prime}$ depends on $\Theta_{0}$. The final version of their test therefore becomes:

$$
y_{t}=\delta_{0}+\boldsymbol{\delta}_{1}^{\prime} \widetilde{\mathbf{x}}_{t}+\boldsymbol{\beta}_{1}^{\prime} \widetilde{\mathbf{x}}_{t} z_{t-d}+\boldsymbol{\beta}_{2}^{\prime} \widetilde{\mathbf{x}}_{t} z_{t-d}^{2}+\boldsymbol{\beta}_{3}^{\prime} \widetilde{\mathbf{x}}_{t} z_{t-d}^{3}+v_{3 t}
$$

\footnotetext{
${ }^{6}$ The exponential autoregressive (EAR) model of Haggan and Ozaki (1981) is a particular case of ESTAR when $\theta_{0}=c=0$ and $z_{t-d}=y_{t-d}$.

7 The term $\frac{1}{2}$ is added here merely for convenience and does not affect the results.

${ }^{8}$ The delay parameter $d$ is usually unknown. Based on Tsay (1989), Teräsvirta (1994) proposes choosing the $d$ that minimizes the p-value of the nonlinearity test.

${ }^{9}$ Also note that the alternative hypothesis will include models other than the STAR.
} 
where the null hypothesis is $H_{0}^{\prime \prime}: \boldsymbol{\beta}_{1}^{\prime}=\boldsymbol{\beta}_{2}^{\prime}=\boldsymbol{\beta}_{3}^{\prime}=\mathbf{0}$. The LM test based on the auxiliary regression (6) is the test adopted by Saikkonen and Luukkonen (1988), Teräsvirta et al. (1994), and Teräsvirta (1994). Their test-statistic is computed by the following procedure: First, estimate (6) under the null hypothesis $H_{0}^{\prime \prime}$ by OLS and calculate the sum of squared residuals, $\mathrm{SSR}_{0}$. Second, using the residuals from the previous step, estimate a model that contains the regressors of (6) to compute the sum of squared residuals $\mathrm{SSR}_{1}$. Then, the statistic $T\left(S S R_{0^{-}}\right.$ $\left.S S R_{1}\right) / S S R_{0}$ will have an asymptotic $\chi^{2}$ distribution with degrees of freedom given by the number of parameter restrictions under $H_{0}^{\prime \prime}$. Those interested in checking the conditions under which these testing results hold can read chapter 6 of Granger and Teräsvirta (1993). In what follows, we refer to this test-statistic as NL3 and make it the focus of our analysis. In practice, the use of the approximation given by the F-distribution is recommended because it has better size and power properties in small samples. An alternative approach is to use the Wald test of Hansen (1996). This procedure approximates the unknown limiting distribution by generating $\mathrm{p}$-values based on simulation methods. ${ }^{10}$

\subsection{Properties of the Taylor series approximation}

The success of the nonlinearity tests and the selection procedures advocated by Teräsvirta (1994) are critically related to the precision of the Taylor series approximation to the transition function. Parsimony requires that this approximation be as economical as possible so as to prevent loss of power of the tests in small samples. However, accuracy of the approximation is paramount to guarantee the consistency of the procedures. Consequently, it is useful to detail the properties of the Taylor series expansion to understand how to best strike a reasonable balance.

Figure 1 illustrates the shapes that the logistic and the exponential transition functions take as a function of the dependent variable, along with the Taylor series approximations to each function. The logistic transition function has a single inflection point and is therefore accurately approximated by a third order series expansion. On the other hand, the exponential transition function has two inflection points. Third order polynomials can only display one inflection point and as Fig. 1 illustrates, the approximation is similar to what one could get with a second order polynomial. A fourth order approximation is also displayed on the bottom right graph of Fig. 1 to illustrate the improvement in accuracy. This additional power allows the approximation to display two inflection points, significantly improving the fit. For the particular example displayed in Fig. 1, the additional fourth order term adds a 30\% improvement in the approximation using the least squares deviations metric.

The rationale for this behavior can be formally explained by computing the derivatives of each type of transition function with respect to the scale parameter. The MacLaurin approximation around $\gamma$ to the transition function defined in (1)

\footnotetext{
10 See Pesaran and Potter (1997) for an application of this technique.
} 

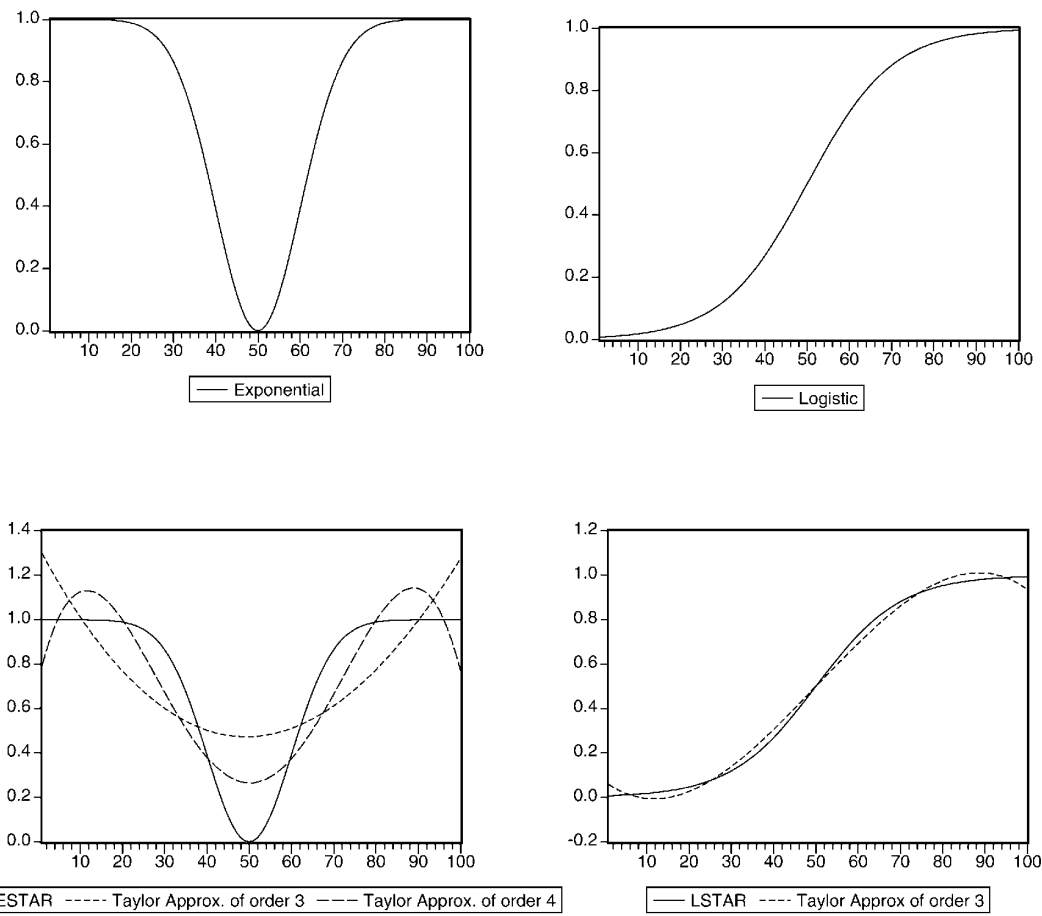

Fig. 1. Smooth transition functions and Taylor series approximations. Notes ESTAR model plot calculated with 100 points ranging from 0 to $1, \gamma=50, c=0.5$. LSTAR model plot calculated with $\gamma=10, c=0.5$. The approximations are computed with OLS regressions. The Taylor approximation of order 3 uses linear, quadratic and cubic terms with the approximation of order 4 in addition includes fourth order powers

is,

$$
\begin{aligned}
F\left(z_{t-d}, \gamma, c\right) & =F\left(z_{t-d}, \gamma=0, c\right)+F_{\gamma}\left(z_{t-d}, \gamma=0, c\right) \gamma+ \\
+F_{\gamma \gamma}\left(z_{t-d}, \gamma\right. & =0, c) \frac{\gamma^{2}}{2 !}+\ldots
\end{aligned}
$$

and in particular for the exponential function of Eq. (2) becomes,

$$
\begin{aligned}
F\left(z_{t-d}, \gamma, c\right)= & \gamma\left(z_{t-d}-c\right)^{2}+\frac{\gamma^{2}}{2 !}\left(z_{t-d}-c\right)^{4}+\ldots+ \\
& \frac{\gamma^{n}}{n !}\left(z_{t-d}-c\right)^{2 n}+\ldots
\end{aligned}
$$

When $c=0$, the MacLaurin polynomial reduces to $F\left(z_{t-d}, \gamma, 0\right)=a_{2} z_{t-d}^{2}+$ $a_{4} z_{t-d}^{4}+\ldots$ where for convenience, the $a_{j}$ represent the appropriate functionals of the original parameters $\gamma$ and $c$. It is clear now why a third order MacLaurin polynomial does not yield a better approximation when $F$ is exponential and symmetrical around $c$ (as in Fig. 1): The cumulative effect of the terms $a_{3} z_{t-d}^{3}$, $a_{5} z_{t-d}^{5}, \ldots, a_{(2 n-1)} z_{t-d}^{2 n-1}, \ldots$ is in general close to zero (exactly zero when $c=0$ ). 
The MacLaurin approximation to the logistic function defined in (3) can be similarly expressed as

$$
\begin{aligned}
F\left(z_{t-d}, \gamma, c\right)= & b_{1}\left(z_{t-d}-c\right)+b_{3}\left(z_{t-d}-c\right)^{3}+\ldots+ \\
& b_{2 n-1}\left(z_{t-d}-c\right)^{2 n-1}+\ldots
\end{aligned}
$$

with the $b_{j}$ representing the corresponding functionals of the parameters $\gamma$ and $c$. Contrary to the exponential function (2), when $c=0$, the approximation only involves the terms $z_{t-d}^{2 n-1}$. It is therefore natural to exploit these distinctive properties in designing tests and decision rules that aim at discriminating between LSTAR and ESTAR models. The next section discusses the manner in which this can be accomplished.

\section{Choosing between LSTAR or ESTAR}

This section exploits an attractive feature of Taylor series approximations to the transition function of a STAR model. We begin by presenting Teräsvirta's (1994) specification procedure and detailing its deficiencies. In response to these concerns, we propose an alternative procedure.

Upon rejecting the null hypothesis of linearity one might consider using a STAR model as a useful non-linear alternative. Teräsvirta (1994) suggests a model selection procedure (which we will denominate TP for short) based on Equation (6). Teräsvirta motivates his procedure by observing the following sequence of nested F-tests:

1. Test the null: $H_{03}: \boldsymbol{\beta}_{3}^{\prime}=\mathbf{0}$ with an F-test $\left(F_{3}\right)$. Teräsvirta notes that in principle, rejection of this null would imply rejection of the ESTAR specification since cubic powers of $z_{t-d}$ in a first order approximation of $F\left(z_{t-d}, \gamma, c\right)$ are 0 when $c=0$.

2. Test the null: $H_{02}: \boldsymbol{\beta}_{2}^{\prime}=\mathbf{0} \mid \boldsymbol{\beta}_{3}^{\prime}=\mathbf{0}$ with an F-test $\left(F_{2}\right)$. Teräsvirta's reasoning is that the $z_{t-d}^{2}$ terms of a first order Taylor series approximation to a logistic function are zero when $c=\Theta_{0}=0$, see (1). However, these terms will be nonzero in the ESTAR case (except in the unlikely case when $\widetilde{\boldsymbol{\Theta}}^{\prime}=\mathbf{0}$ ). Failure to reject this null is taken as evidence in favor of a LSTAR model. Nevertheless, rejection of $H_{02}$ is not very informative one way or the other.

3. Test the null: $H_{01}: \boldsymbol{\beta}_{1}^{\prime}=\mathbf{0} \mid \boldsymbol{\beta}_{2}^{\prime}=\boldsymbol{\beta}_{3}^{\prime}=\mathbf{0}$ with an F-test $\left(F_{1}\right)$. Following Teräsvirta, failing to reject $H_{01}$ after rejecting $H_{02}$ points to an ESTAR model. On the other hand, rejecting $H_{01}$ after failing to reject $H_{02}$ supports the choice of LSTAR.

Based on these tests, Teräsvirta's rule consists on noting which hypotheses are rejected and then comparing the relative strengths of the rejections. If the model is LSTAR, typically $H_{01}$ and $H_{03}$ are rejected more strongly than $H_{02}$. Therefore, Teräsvirta proposed to select an ESTAR specification if the p-value of $F_{2}$ is the smallest of $F_{1}, F_{2}, F_{3}$, otherwise the LSTAR alternative is preferred. 
Teräsvirta recognizes that this procedure might cause problems. For example, even if the model is ESTAR, $H_{03}$ might be rejected since $\boldsymbol{\beta}_{3}^{\prime}=\mathbf{0}$ only if $c=$ $\Theta_{0}=0$ in (1). These concerns and others are best illustrated by analyzing the terms of the Taylor series expansion for each non-linear state in (1):

$$
\Theta^{\prime} \widetilde{\mathbf{x}}_{t}\left[1-\exp \left\{-\gamma\left(z_{t-d}-c\right)^{2}\right\}\right] \simeq \boldsymbol{\Psi}_{2}^{\prime} \widetilde{\mathbf{x}}_{t}\left(z_{t-d}-c\right)^{2}+\boldsymbol{\Psi}_{4}^{\prime} \widetilde{\mathbf{x}}_{t}\left(z_{t-d}-c\right)^{4}
$$

for the first four terms of the MacLaurin polynomial of the exponential function, where we have used the result that the terms $\left(z_{t-d}-c\right)^{2 n-1}$ in said polynomial vanish. Similarly,

$$
\boldsymbol{\Theta}^{\prime} \widetilde{\mathbf{x}}_{t}\left[\left\{1+\exp \left(-\gamma\left(z_{t-d}-c\right)\right)\right\}^{-1}-\frac{1}{2}\right] \simeq \boldsymbol{\Psi}_{1}^{\prime} \widetilde{\mathbf{x}}_{t}\left(z_{t-d}-c\right)+\boldsymbol{\Psi}_{3}^{\prime} \widetilde{\mathbf{x}}_{t}\left(z_{t-d}-c\right)^{3}
$$

using also the first four terms of the MacLaurin polynomial of the logistic function. In this case we use the result that the terms $\left(z_{t-d}-c\right)^{2 n}$ vanish. The shorthand notation $\boldsymbol{\Psi}_{i}^{\prime}$ for $i=1,2,3,4$ collects the parameters associated with each of the terms rather than expressing complicated formulas of parameters that can not be identified in a regression model.

Teräsvirta's concerns can now be easily understood by inspecting (9). Whenever $c, \pi_{0}$ and/or $\theta_{0}$ are non-zero, expansion of $\widetilde{\mathbf{x}}_{t}\left(z_{t-d}-c\right)^{4}$ yields non-zero $\widetilde{\mathbf{x}}_{t} z_{t-d}^{3}$ terms. In addition, when the variance of the error term is "large" the distribution of the data into each state around the threshold $c$ tends to be asymmetric. As a result, the null hypothesis $H_{02}: \boldsymbol{\beta}_{2}^{\prime}=\mathbf{0} \mid \boldsymbol{\beta}_{3}^{\prime}=\mathbf{0}$ does not discriminate between a LSTAR with $c \neq 0$ and an ESTAR in general.

An additional source of complications lies in the design of the rule itself. For example, if the true model is LSTAR, it is unclear that by conditioning on the cubic terms to be zero (that is, restricting $\boldsymbol{\beta}_{3}^{\prime}=\mathbf{0}$ in (1)), the joint significance of the square terms, $\widetilde{\mathbf{x}}_{t} z_{t-d}^{2}$ (from (10), these are non-zero since $c \neq 0$ ) will be also zero. These terms are now left to approximate the transition function - an approximation that the cubic terms presumably were successfully capturing.

Inspection of Eqs. (9) and (10) provides clues on how to correct the deficiencies of TP. Consider the following example. Suppose $c=0$. Based on (10), it is clear that if the model is LSTAR, the terms $\widetilde{\mathbf{x}}_{t} z_{t-d}^{j}$ for $j=2,4$, are zero (i.e. $\boldsymbol{\beta}_{2}^{\prime}=\boldsymbol{\beta}_{4}^{\prime}=\mathbf{0}$ in (12) below). Alternatively, if the model is ESTAR, based on (9), the terms $\widetilde{\mathbf{x}}_{t} z_{t-d}^{j}$ for $j=1,3$, are zero (i.e. $\boldsymbol{\beta}_{1}^{\prime}=\boldsymbol{\beta}_{3}^{\prime}=\mathbf{0}$ in (12) below). Therefore, the behavior of the MacLaurin polynomial for the transition function suggests that the regression (6) could be modified by adding an additional term as follows,

$$
\begin{aligned}
y_{t}= & \pi_{0}+\boldsymbol{\pi}_{1}^{\prime} \widetilde{\mathbf{x}}_{t}+\boldsymbol{\Psi}_{1}^{\prime} \widetilde{\mathbf{x}}_{t}\left(z_{t-d}-c\right)+\boldsymbol{\Psi}_{2}^{\prime} \widetilde{\mathbf{x}}_{t}\left(z_{t-d}-c\right)^{2}+ \\
& \boldsymbol{\Psi}_{3}^{\prime} \widetilde{\mathbf{x}}_{t}\left(z_{t-d}-c\right)^{3}+\boldsymbol{\Psi}_{4}^{\prime} \widetilde{\mathbf{x}}_{t}\left(z_{t-d}-c\right)^{4}+v_{4 t}
\end{aligned}
$$

or, more specifically, in terms of observable parameters,

$$
y_{t}=\delta_{0}+\boldsymbol{\delta}_{1}^{\prime} \widetilde{\mathbf{x}}_{t}+\boldsymbol{\beta}_{1}^{\prime} \widetilde{\mathbf{x}}_{t} z_{t-d}+\boldsymbol{\beta}_{2}^{\prime} \widetilde{\mathbf{x}}_{t} z_{t-d}^{2}+\boldsymbol{\beta}_{3}^{\prime} \widetilde{\mathbf{x}}_{t} z_{t-d}^{3}+\boldsymbol{\beta}_{4}^{\prime} \widetilde{\mathbf{x}}_{t} z_{t-d}^{4}+v_{4 t}
$$


This would require augmenting the nonlinearity test (6) with the additional terms captured in $\boldsymbol{\beta}_{4}^{\prime} \widetilde{\mathbf{x}}_{t} z_{t-d}^{4}$. We delay the discussion of this alternative nonlinearity test which we denominate NL4 for future reference. Here we concentrate on how to use the additional information to select the appropriate nonlinear STAR model.

We propose the following alternative selection procedure, which we denominate EJP (Escribano-Jordá):

1. Test for nonlinearity and if linearity is rejected, then

2. test the null: $H_{0 L}: \boldsymbol{\beta}_{2}^{\prime}=\boldsymbol{\beta}_{4}^{\prime}=\mathbf{0}$ with an F-test $\left(F_{L}\right)$ and

3. test the null: $H_{0 E}: \boldsymbol{\beta}_{1}^{\prime}=\boldsymbol{\beta}_{3}^{\prime}=\mathbf{0}$ with an F-test $\left(F_{E}\right)$.

4. If the minimum p-value corresponds to $F_{E}$, select LSTAR, otherwise select ESTAR.

This 4-step selection procedure is clearly consistent when $c=0$ following the motivation in our example. However, when $c \neq 0$, EJP is still effective since we are comparing the joint significance of linear and cubic terms relative to the joint significance of quadratic and fourth order terms, without conditioning on other parameters being zero.

Aside from its simplicity and effectiveness, EJP could be transformed to provide information about non-zero thresholds, $c$. Remember that linear and cubic terms are exactly zero when $c=0$ and the model is ESTAR while quadratic and fourth order terms are exactly zero when $c=0$ and the model is LSTAR.

The procedure to select $\mathrm{c}$ is called the c-procedure and works as follows:

1. Test for nonlinearity and if linearity is rejected, then

2. rejecting $H_{0 E}$ with $F_{E}$ and failing to reject $H_{0 L}$ with $F_{L}$ suggests an LSTAR model with $c=0$

3. rejecting $H_{0 L}$ with $F_{L}$ and failing to reject $H_{0 E}$ with $F_{E}$ suggests a ESTAR model with $c=0$.

This simple c-procedure is useful to set $c=0$ as a good starting value in the parametric nonlinear estimation when linearity was clearly rejected. In other borderline cases the results of the c-procedure could be informative but care must be taken interpreting the results as will become clear later on in the Monte Carlo simulation experiments.

\subsection{Modifying the nonlinearity test}

The power of the nonlinearity test NL3 depends crucially on the quality of the Taylor series approximation to the transition function. We have seen that the MacLaurin polynomials of the transition function indicate that when this transition function is exponential, one would require terms of the form $\widetilde{\mathbf{x}}_{t} z_{t-d}^{4}$ to capture the two inflection points discussed above. Additionally, we have seen that these terms can be exploited to improve on the selection procedure. The key question that needs to be answered is whether the additional terms add sufficient 

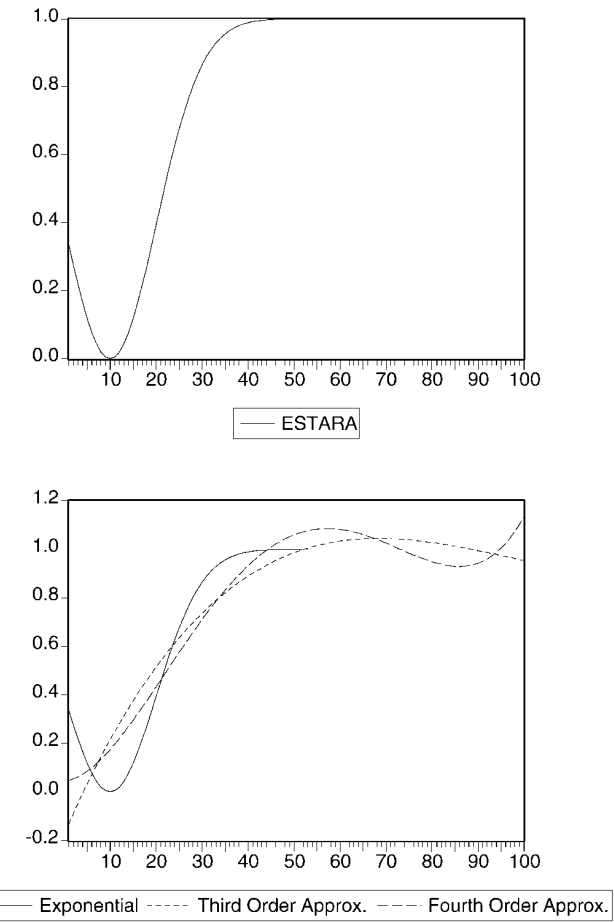

Fig. 2. Assymmetric ESTAR model: Third and fourth order Taylor serie approximations. Notes ESTAR model constructed with 100 points between 0 and $1, \gamma=50, c=0.1$. The approximations are computed with OLS regressions. The Taylor approximation of order 3 uses linear, quadratic and cubic terms while the approximation of order 4 in addition includes fourth order powers

precision in the approximation to justify augmenting the nonlinearity test based on (6) with these additional regressors.

This is a matter that can only be answered empirically, on a case by case basis. In the example depicted in Fig. 1 these additional regressors cause the SIC to drop from 0.094662 to -0.875749 (in a simple OLS regression of the data generated with the exponential transformation on the levels, squares, third and fourth powers of the data), a remarkable change. However, as Fig. 2 shows, asymmetric ESTAR models maybe properly approximated with third order expansions (the improvement in SIC is now only from -1.267067 to -1.598299$)$. In any case, the selection procedure EJP introduced above, requires that the auxiliary regression be specified as,

$$
y_{t}=\delta_{0}+\boldsymbol{\delta}_{1}^{\prime} \widetilde{\mathbf{x}}_{t}+\boldsymbol{\beta}_{1}^{\prime} \widetilde{\mathbf{x}}_{t} z_{t-d}+\boldsymbol{\beta}_{2}^{\prime} \widetilde{\mathbf{x}}_{t} z_{t-d}^{2}+\boldsymbol{\beta}_{3}^{\prime} \widetilde{\mathbf{x}}_{t} z_{t-d}^{3}+\boldsymbol{\beta}_{4}^{\prime} \widetilde{\mathbf{x}}_{t} z_{t-d}^{4}+v_{4 t}
$$

where the null hypothesis of linearity now becomes $H_{0}^{\prime \prime \prime}: \boldsymbol{\beta}_{1}^{\prime}=\boldsymbol{\beta}_{2}^{\prime}=\boldsymbol{\beta}_{3}^{\prime}=\boldsymbol{\beta}_{4}^{\prime}=\mathbf{0}$. The LM test-statistic of this joint hypothesis is called NL4. ${ }^{11}$ Compared to NL3, NL4 requires $p$ extra regressors in the auxiliary regression. Testing nonlinearity

\footnotetext{
11 When $z_{t-d}=y_{t-d}$, this test is similar in spirit to a high order RESET test, see Ramsey (1969).
} 
in practice involves several important steps such as choice of lag length of the AR model and choice of delay parameter $d$. These are well documented in Teräsvirta (1994).

In situations where lack of parsimony is of concern, a simplified version of NL4 based on the results in Luukkonen et al. (1988a) could be considered. Parallel to their augmented first order procedure, the auxiliary regression in the STAR case becomes:

$$
y_{t}=\delta_{0}+\boldsymbol{\delta}_{1}^{\prime} \widetilde{\mathbf{x}}_{t}+\boldsymbol{\beta}_{1}^{\prime} \widetilde{\mathbf{x}}_{t} z_{t-d}+\beta_{2}^{*} z_{t-d}^{3}+\beta_{3}^{*} z_{t-d}^{4}+\beta_{4}^{*} z_{t-d}^{5}+v_{4 t}^{*} .
$$

The corresponding null hypothesis of linearity is $H_{0}^{I V}: \boldsymbol{\beta}_{1}^{\prime}=\mathbf{0} ; \beta_{2}^{*}=\beta_{3}^{*}=$ $\beta_{4}^{*}=0$. We term this test simplified-NL test. Care should be taken in using this simplification since in several cases of ESTAR models certain parameters are zero.

\section{Monte Carlo experiments}

This section provides Monte Carlo evidence in support of our recommended decision procedure, EJP relative to the selection procedures TP and GTP - which stands for "generalized" TP and is designed to take advantage of the fact that the nonlinearity auxiliary regression contains extra terms. ${ }^{12}$ Additional evidence is provided to compare the nonlinearity tests NL3, and NL4. The models simulated in this study are taken from Luukkonen et al. (1988a,b) and Teräsvirta (1994). Each experiment is replicated 1,000 times. The first 100 observations of each series are disregarded to avoid initialization problems. ${ }^{13}$

\subsection{Selection frequencies of the EJP selection procedure}

Tables 1 and 2 summarize the simulation results with ESTAR and LSTAR data generating processes and compare the accuracy of EJP and TP in selecting the correct model. The correct selection rate is reported as a percentage of those replications for which linearity was first rejected at the conventional 95\% confidence level. We explore the robustness of the selection procedures by considering additional examples with non zero thresholds (i.e. $c \neq 0$ ) that make the model asymmetric (as in Fig. 2) and misspecification of the lag structure.

The first panel of Table 1 illustrates that, when the DGP is ESTAR, EJP uniformly outperforms TP, particularly when the threshold is zero. Higher values of the threshold make the model more asymmetric.. This has the effect of making the ESTAR and LSTAR specifications observationally similar and in some cases, it makes the model appear linear with a few outliers. This may explain the poor performance of TP in the selecting ESTAR in the extreme case $c=1.5$.

\footnotetext{
12 The steps involved require the sequential tests, $H_{0}: \boldsymbol{\beta}_{4}^{\prime}=0, H_{0}: \boldsymbol{\beta}_{3}^{\prime}=0 \mid \boldsymbol{\beta}_{4}^{\prime}=0, H_{0}: \boldsymbol{\beta}_{2}^{\prime}=$ $0 \mid \boldsymbol{\beta}_{3}^{\prime}=\boldsymbol{\beta}_{4}^{\prime}=0$, and $H_{0}: \boldsymbol{\beta}_{1}^{\prime}=0 \mid \boldsymbol{\beta}_{4}^{\prime}=\boldsymbol{\beta}_{3}^{\prime}=\boldsymbol{\beta}_{2}^{\prime}=0$.

${ }^{13}$ Extensive Monte Carlo evidence and detailed description of the experiments is available in Escribano and Jorda (1997).
} 
Table 1. Selection frequencies of the procedures

\begin{tabular}{llllllll}
\hline & & $\pi_{0}=0$ & \multicolumn{5}{c}{$\pi_{0}=0.3$} \\
\hline Threshold & Sample & TP & EJP & NL4 Power & TP & EJP & NL4 Power \\
C $=0$ & 50 & 0.686 & 0.859 & 0.156 & 0.523 & 0.788 & 0.193 \\
& 100 & 0.840 & 0.909 & & 0.601 & 0.894 & \\
& 200 & 0.937 & 0.985 & & 0.678 & 0.971 & \\
$\mathrm{C}=0.3$ & 50 & 0.609 & 0.731 & 0.156 & 0.640 & 0.690 & 0.200 \\
& 100 & 0.805 & 0.781 & & 0.714 & 0.859 & \\
& 200 & 0.899 & 0.876 & & 0.837 & 0.910 & \\
$\mathrm{C}=1.5$ & 50 & 0.132 & 0.212 & 0.349 & 0.120 & 0.357 & 0.350 \\
& 100 & 0.057 & 0.170 & & 0.097 & 0.301 & \\
& 200 & 0.012 & 0.089 & & 0.057 & 0.246 & \\
\hline
\end{tabular}

Relative frequencies of correct specification of ESTAR models (as a proportion of the replications for which linearity is rejected at a 95\% confidence level using NL4). Table 4, p. 172, Luukkonen et al. (1988b).

$1=100 \%$ accuracy selecting the correct model, $0=0 \%$ accuracy. DGP:

$$
y_{1}=\pi_{0}+0.3 y_{t-1}-\left(0.9 y_{t-1}\right)\left\{1-\exp \left(-\left(y_{t-1}-c\right)^{2}\right)\right\}+\varepsilon_{t} \quad \varepsilon_{t} \sim N(0,0.36)
$$

\begin{tabular}{|c|c|c|c|c|c|c|c|c|c|}
\hline & & $\pi_{1}=-0.5$ & & & $\pi_{1}=0$ & & & & \\
\hline Sample & $\theta_{1}$ & Threshold & TP & $\begin{array}{l}\text { EJP } \\
\text { Power }\end{array}$ & NL4- & $\theta_{1}$ & $\begin{array}{l}\text { TP } \\
\text { Power }\end{array}$ & EJP & NL4- \\
\hline \multirow[t]{12}{*}{50} & \multirow[t]{3}{*}{-0.4} & $\mathrm{C}=0$ & 0.793 & 0.683 & 0.082 & \multirow[t]{3}{*}{0.5} & 0.914 & 0.797 & 0.187 \\
\hline & & $C=10$ & 0.663 & 0.542 & 0.083 & & 0.808 & 0.717 & 0.099 \\
\hline & & $\mathrm{C}=15$ & 0.762 & 0.492 & 0.063 & & 0.789 & 0.615 & 0.052 \\
\hline & \multirow[t]{3}{*}{0.5} & $\mathrm{C}=0$ & 0.914 & 0.797 & 0.187 & \multirow[t]{3}{*}{-0.5} & 0.823 & 0.735 & 0.113 \\
\hline & & $\mathrm{C}=10$ & 0.808 & 0.717 & 0.099 & & 0.703 & 0.595 & 0.111 \\
\hline & & $C=15$ & 0.788 & 0.615 & 0.052 & & 0.776 & 0.507 & 0.067 \\
\hline & \multirow[t]{3}{*}{1} & $\mathrm{C}=0$ & 0.994 & 0.979 & 0.875 & \multirow[t]{3}{*}{-1} & 0.898 & 0.931 & 0.305 \\
\hline & & $\mathrm{C}=10$ & 0.883 & 0.833 & 0.480 & & 0.810 & 0.593 & 0.300 \\
\hline & & $C=15$ & 0.837 & 0.618 & 0.123 & & 0.744 & 0.511 & 0.129 \\
\hline & \multirow[t]{3}{*}{1.5} & $\mathrm{C}=0$ & 0.999 & 0.999 & 0.998 & \multirow[t]{3}{*}{-1.4} & 0.547 & 0.981 & 0.464 \\
\hline & & $C=10$ & 0.948 & 0.968 & 0.857 & & 0.848 & 0.615 & 0.538 \\
\hline & & $C=15$ & 0.857 & 0.728 & 0.265 & & 0.726 & 0.511 & 0.186 \\
\hline \multirow[t]{4}{*}{100} & -0.4 & $\mathrm{C}=0$ & 0.459 & 0.811 & 0.122 & 0.5 & 0.919 & 0.860 & 0.272 \\
\hline & 0.5 & & 0.811 & 0.724 & 0.127 & -0.5 & 0.898 & 0.814 & 0.118 \\
\hline & 1 & & 0.952 & 0.936 & 0.498 & -1 & 0.941 & 0.935 & 0.491 \\
\hline & 1.5 & & 0.963 & 0.978 & 0.883 & -1.4 & 0.594 & 0.978 & 0.744 \\
\hline
\end{tabular}

Relative frequencies of correct specification of LSTAR model (as a proportion of replications for which linearity is rejected at a 95\% confidence level). Fig. 2, p. 496, Luukkonen et al. (1988a). $1=100 \%$ accuracy selecting the correct model, $0=0 \%$ accuracy. DGP:

$$
y_{1}=\pi_{1} y_{t-1}-\left(\theta_{1} y_{t-1}\right)\left(1+\exp \left\{-0.5\left(y_{t-1}-c\right)\right\}\right)^{-1}+\varepsilon_{1} \quad \varepsilon_{1} \sim N(0,25.0)
$$


Table 2.

Data generated from models 4.1-4.2 and 4.6, g. 210-211, in Teräsvirta (1994). DGP:

$$
\begin{array}{ccc}
y_{1}=1.8 y_{t-1}-1.06 y_{t-2}+\left(\pi_{20}-0.9 y_{t-1}+0.795 y_{t-2}\right) F\left(y_{t-1}\right)+u_{t} ; & u_{t} \sim N(0,0.04) \\
F\left(y_{t-1}\right) & =\left(1+\exp \left\{-\gamma\left(y_{t-1}-c\right)\right\}\right)^{-1}, \quad \text { LSTAR; } \\
F\left(y_{t-1}\right) & =1-\exp \left\{-1000\left(y_{t-1}-c\right)^{2}\right\}, \quad \text { ESTAR }
\end{array}
$$

- Size properties

Sample size $=100$

\begin{tabular}{lll}
\hline Lags & NL4 - Size & NL3 - Size \\
\hline 2 & 0.043 & 0.043 \\
3 & 0.042 & 0.052 \\
4 & 0.045 & 0.044 \\
\hline
\end{tabular}

- Sensitivity of Selection Frequency and Power to the lag structure Correct lag length is $\mathrm{p}=2, \mathrm{~d}=1$.

Model: ESTAR

Sample size $=100$

\begin{tabular}{lllllll}
\hline & & & & 3 & Power \\
\hline & Lags & TP & GTP & EJP & NL4 & NL3 \\
\hline $\mathrm{C}=0, \pi_{20}=0$ & 2 & 0.885 & 0.922 & 0.971 & 0.729 & 0.608 \\
& 3 & 0.849 & 0.901 & 0.956 & 0.615 & 0.507 \\
$\mathrm{C}=0, \pi_{20}=0.02$ & 2 & 0.809 & 0.869 & 0.959 & 0.518 & 0.450 \\
& 3 & 0.673 & 0.698 & 0.671 & 0.838 & 0.851 \\
$\mathrm{C}=0.02$, & 4 & 0.692 & 0.717 & 0.666 & 0.769 & 0.780 \\
$\pi_{20}=0.04$ & 2 & 0.488 & 0.595 & 0.682 & 0.702 & 0.724 \\
& 3 & 0.493 & 0.574 & 0.350 & 0.606 & 0.574 \\
& 4 & 0.489 & 0.549 & 0.356 & 0.556 & 0.528 \\
& & & & & & 0.485 \\
\hline
\end{tabular}

Model: LSTAR

Sample size $=100$

\begin{tabular}{lllllll}
\hline & & & & 3 & Power \\
\hline & Lags & TP & GTP & EJP & NL4 & NL3 \\
\hline $\mathrm{C}=0, \pi_{20}=0$ & 2 & 0.975 & 0.965 & 0.998 & 0.950 & 0.962 \\
& 3 & 0.970 & 0.962 & 0.999 & 0.915 & 0.937 \\
$\mathrm{C}=0, \pi_{20}=0.02$ & 2 & 0.962 & 0.953 & 0.993 & 0.878 & 0.905 \\
& 3 & 0.932 & 0.909 & 0.907 & 0.636 & 0.660 \\
$\mathrm{C}=0.02$, & 4 & 0.923 & 0.878 & 0.895 & 0.588 & 0.606 \\
$\pi_{20}=0.04$ & 2 & 0.859 & 0.827 & 0.893 & 0.533 & 0.567 \\
& 3 & 0.848 & 0.790 & 0.514 & 0.156 & 0.138 \\
& 4 & 0.838 & 0.752 & 0.599 & 0.137 & 0.140 \\
\hline
\end{tabular}


Table 2. (continued)

Sample size $=300$

\begin{tabular}{lllllll}
\hline & & & & & Power \\
\hline Model & Lags & TP & GTP & EJP & NL4 & NL3 \\
\hline \multirow{2}{*}{ ESTAR } & $\mathrm{C}=0, \pi_{20}=0$ & 0983 & 0.989 & 1.000 & 0.999 & 0.983 \\
& $\mathrm{C}=0, \pi_{20}=0.02$ & 0.876 & 0.883 & 0.850 & 1.000 & 1.000 \\
& $\mathrm{C}=0.02, \pi_{20}=0.04$ & 0332 & 0.379 & 0.569 & 0.982 & 0.975 \\
LSTAR & $\mathrm{C}=0, \pi_{20}=0$ & 1.000 & 1.000 & 1.000 & 1.000 & 1.000 \\
& $\mathrm{C}=0, \pi_{20}=0.02$ & 0998 & 0.996 & 0.985 & 0.993 & 0.991 \\
& $\mathrm{C}=0.02, \pi_{20}=0.04$ & 0956 & 0.925 & 0.671 & 0.386 & 0.378 \\
\hline
\end{tabular}

The second panel of Table 1 concentrates on LSTAR DGPs. Both procedures work relatively well, with TP performing marginally better. However, EJP tends to outperform TP when the nonlinearity is stronger (by stronger we mean the nonlinearity test has more power) and conversely when the nonlinearity is harder to detect. For instance, when $\pi_{1}=0.5$ and $\theta_{1}=-1.4$, and $c=0$, EJP correctly selects LSTAR $98 \%$ of the time while TP selects it only $55 \%$ of the time. When the threshold is significantly different from 0 (in the simulations, $c=15$ ) then TP seems to perform better than EJP. However, with $c=15$, the model becomes linear with a few outliers (note that the power of the nonlinearity test also drops significantly from $46 \%$ to $19 \%$ ). With larger sample sizes (100 in our exercise), both procedures do reasonably well. It is important to note that when the data is evenly distributed between the two regimes (although possibly asymmetrically), the results in Table 1 indicate that EJP outperforms TP. In practice, these are likely to be the relevant situations in which to use STAR models. In certain cases it is hard to distinguish nonlinear models from a conventional linear model with a few outliers. This topic is beyond the scope of this paper but it is thoroughly investigated in Escribano et al. (1998) and Van Dijk et al. (1998).

Table 2 investigates the sensitivity of the selection procedures to incorrect lag length specification. Both ESTAR and LSTAR models are simulated with an AR(2) model borrowed from Teräsvirta (1994). Similarly to Table 1, EJP performs better than TP for both ESTAR and LSTAR when $c=0$ although both do better when the data is not clustered in one of the regimes - for this exercise, cases with either $c$ and/or $\pi_{20}$ different from zero. In general, both the EJP and TP rules seem rather insensitive to overparametrization of the auxiliary regressions (e.g. including four lags when only two are needed). Table 2 (continued) also highlights that TP can be "inconsistent" as we discussed above. In particular, when $c=0.02$ and $\pi_{20}=0.04$, note that the correct selection of the ESTAR model drops for TP from $49 \%$ to $33 \%$ as the sample size increases from 100 to 300 observations. By contrast, EJPs selection frequency improves from 35\% to $57 \%$ with the increase in sample size. 


\subsection{Power and size properties of the nonlinearity tests}

We provide evidence of the performance and the sensitivity of the nonlinearity tests to incorrect specifications of the lag length for the examples in Table 2 by comparing the NL3 to the NL4 test. Other simulations are included in Escribano and Jordá $(1997,1999)$. The size of the tests is relatively insensitive to overparametrization of the $\mathrm{AR}(\mathrm{p})$ lags and the power loss is in general, not very important. The simulations indicate that with large sample sizes (in our study 300 observations), there is little difference between NL3 and NL4. Both tests detect non-linearity adequately, with the power approximating 1 in most cases. However, for smaller sample sizes, (in our study 100 observations), NL4 has higher power than NL3 when the true model is ESTAR (in particular when both $c$ and $\pi_{20}$ are zero or both nonzero). When the true model is LSTAR, NL4 does not significantly loose power with respect to NL3. These results are encouraging for the NL4 test for two reasons: (1) when the model is LSTAR we showed that the fourth order powers are zero in the specific case $c=0$, and (2) as Fig. 2 illustrates, although asymmetric ESTAR functions (close substitutes to LSTAR) appear well approximated by third order powers, the contribution of fourth order powers is still significant. Compare the power of NL3 and NL4 of Table 2 for sample sizes of 300 observations from ESTAR and LSTAR models.

\section{Teräsvirta and Anderson (1992) revisited}

Teräsvirta and Anderson (1992) analyze the dynamic properties of industrial production indices of thirteen OECD countries and an European aggregate using STAR models. The data is quarterly, seasonally unadjusted,,${ }^{14}$ and spans from 1960:I to 1986:IV. ${ }^{15}$ This section replicates nonlinearity testing and model selection with our alternatives, NL4 and EJP. Our goal is to compare the performance of the new procedures in practice. Table 4 reports p-values of nonlinearity tests, delay parameter choice, and STAR model selection for those countries in which nonlinearities were detected either by NL3 or NL4. ${ }^{16}$

NL3 and NL4 obtain their minimum p-values for the same choice of delay parameter, $d$, except in the case of the U.S.A. While the results of both tests are similar, NL4 fails to reject linearity at the usual 5\% level for 3 countries. ${ }^{17}$ With regard to EJP, the same models are selected as with TP except for Austria and Sweden. In the case of Japan, Teräsvirta and Anderson (1992) report that choosing between models (LSTAR or ESTAR) was hard with TP and hence estimated both specifications. The final model they selected on the basis of fit was an ESTAR - a choice that EJP selects unequivocally.

\footnotetext{
${ }^{14}$ They make the series approximately stationary by fourth lag differencing $\left(\mathrm{x}_{t}-\mathrm{x}_{t-4}\right)$.

15 Source: OECD Main Economic Indicators.

${ }^{16}$ French and Italian indices were adjusted for strikes and other anomalies and are therefore not considered here.

17 A sample of 104 observations and the extra regressors required by NL4 probably justify this result.
} 
Table 3. Linearity testing, determining the delay parameter and selecting between LSTAR and ESTAR models

\begin{tabular}{lcrcrrr}
\hline Country & $\begin{array}{c}\text { Max. Lag } \\
\text { (AIC) }\end{array}$ & $\begin{array}{r}\text { P-value } \\
\text { NL3 }\end{array}$ & $\begin{array}{r}\text { P-value } \\
\text { NL4 }\end{array}$ & $\begin{array}{r}\text { Delay } \\
\text { Parameter }\end{array}$ & $\begin{array}{r}\text { TP } \\
\text { Choice }\end{array}$ & $\begin{array}{r}\text { EJP } \\
\text { Choice }\end{array}$ \\
\hline Austria & 5 & 0.010 & 0.033 & 1 & LSTAR & ESTAR \\
Belgium & 5 & 0.050 & 0.259 & 1 & LSTAR & LSTAR \\
Japan & 5 & 0.000 & 0.000 & 1 & $?$ & ESTAR \\
Norway & 8 & 0.031 & 0.200 & 5 & LSTAR & LSTAR \\
Sweden & 5 & 0.015 & 0.040 & 3 & LSTAR & ESTAR \\
U.K. & 8 & 0.047 & 0.192 & 4 & ESTAR & ESTAR \\
U.S.A. & 6 & 0.006 & $0.054 / 0: 016$ & $3 / 5^{*}$ & LSTAR & LSTAR \\
EUR & 9 & 0.015 & 0.043 & 3 & ESTAR & ESTAR \\
\hline
\end{tabular}

Note For U.S.A. NL4 minimum p-value was for $\mathrm{d}=5$.

Table 4. Summary statistics for STAR model estimation: Austria and Sweden

\begin{tabular}{lcrlrr}
\hline & Austria & & & Sweden \\
\hline $\begin{array}{l}\text { Summary } \\
\text { Statistics }\end{array}$ & LSTAR & ESTAR & $\begin{array}{l}\text { Summary } \\
\text { Statistics }\end{array}$ & LSTAR & ESTAR \\
\hline$R$-Squared & 0.7079 & 0.6778 & R-Squared & 0.7251 & 0.7311 \\
Adj. $R 2$ & 0.6819 & 0.6607 & Adj. $R 2$ & 0.7039 & 0.7104 \\
SSR & 0.0503 & 0.0554 & SSR & 0.0538 & 0.0526 \\
AIC & -7.4036 & -7.3770 & AIC & -7.3560 & -7.3781 \\
SIC & -7.1677 & -7.2207 & SIC & -7.1463 & -7.1684 \\
Durbin-Watson & 2.3350 & 2.0845 & Durbin-Watson & 1.8476 & 2.0132 \\
\# of params. & 9 & 6 & \# of params. & 8 & 8 \\
\hline
\end{tabular}

We estimated both LSTAR and ESTAR specifications for Austria and Sweden to determine which specification fitted best. The estimates for Austria are harder to compare since the final models have a different number of parameters - Schwarz's information criterion (SIC) favors the ESTAR specification while Akaike's (AIC) favors the LSTAR specification. However, in the case of Sweden, the final models have the same number of parameters. The preferred specification is the ESTAR (which was selected by EJP but not by TP) with a better fit overall than its LSTAR counterpart. Of course, the true model is unknown. The value of this exercise was to check what specification seemed to work best and what specification test led us to it.

\section{Conclusion}

This paper proposes a selection procedure (EJP) to choose between logistic STAR (LSTAR) and exponential STAR (ESTAR) specifications when the alternative hypothesis to the null of linearity is a nonlinear STAR type of model. This new decision rule is simpler, more intuitive, and has better properties than the usual TP rule, proposed by Teräsvirta(1994). This is particularly relevant when the true model is ESTAR, which is the most common parameterization used in 
empirical models of the business cycles of the USA and Europe. That conclusion is clearly reached from our simulations when the nonlinearity is strong and when the threshold (c) is cero. However, we simulated other nonlinear models (STAR with c different than 0) to check the robustness of both decision rules to extreme asymmetries. The resulting nonlinear asymmetric models are observationally equivalent to linear models with few outliers and therefore, one should expect that both decision rules perform badly (see Escribano et al. (1998) for a robust analysis of those cases). Along the way, we have provided practical guidelines regarding nonlinearity testing when the data are mainly in one of the regimes (asymmetric ESTAR or LSTAR) and when there is uncertainty about the lag length of the autoregressive part . In support of our claims, we conducted Monte-Carlo simulations and applied our procedures to the industrial production indices of thirteen OECD countries and to a European aggregate. All these hypothesis testing procedures discussed here could easily be generalized to Smooth Transition Regression models and to other multivariate models. ${ }^{18}$

\section{References}

Davies, R. B. (1977) Hypothesis Testing When a Nuisance Parameter is Present Only Under the Alternative. Biometrika 64: 247-254

Escribano, A., Franses, P. H., van Dijk, D. (1998) Nonlinearities and Outliers: Robust Specification of STAR Models. Report 9832/A, Econometric Institute, Erasmus University of Rotterdam

Escribano, A., Jordá, O. (1999) Improved Testing and Specification of Smooth Transition Regression Models. In: Rothman, P. (ed.) Nonlinear Time Series Analysis of Economic and Financial Data. Kluwer Academic Publishers, Dordrecht

Escribano, A., Jordá, O. (1997) Testing Nonlinearity: Decision Rules for Selecting Between Logistic and Exponential STAR Models. Working Paper 97-63, Universidad Carlos III de Madrid

Gallant A. R. (1987) Nonlinear Statistical Models. Wiley, New York

Granger, C. W. J., Andersen, A. P. (1978) An Introduction to Bilinear Time Series Models. Vandenhoeck and Ruprecht, Göttingen

Granger, C. W. J., Teräsvirta, T. (1993) Modeling Nonlinear Economic Relationships. Oxford University Press, New York

Haggan, V., Ozaki, T. (1981) Modelling Nonlinear Random Vibrations Using an AmplitudeDependent Autoregressive Time Series Model. Biometrika 68: 189-196

Hamilton, J. D. (1989) A New Approach to the Economic Analysis of Non Stationary Time Series and the Business Cycle. Econometrica 57: 357-384

Hansen, B. (1996) Inference when a Nuisance Parameter is not Identified under the Null Hypothesis. Econometrica 64: 413-430

Luukkonen, R., Saikkonen, P. and Teräsvirta, T. (1988a) Testing Linearity Against Smooth Transition Autoregressive Models. Biometrika 75: 491-499

Luukkonen, R., Saikkonen, P., Teräsvirta, T. (1988b) Testing Linearity in Univariate Time Series Models. Scandinavian Journal of Statistics 15: 161-175

Mira S., Escribano, A. (2000) Nonlinear Time Series Models: Consistency and Asymptotic Normality of NLS Under New Conditions. In: Barnett, W. A., Hendry, D. F., Hylleberg, S., Teräsvirta, T., Tjøstheim, D., Würtz, A. (eds.) Nonlinear Econometric Modelling in Time Series Analysis. Cambridge University Press, Cambridge

Neftçi, S. N. (1984) Are Economic Time Series Asymmetric over the Business Cycle? Journal of Political Economy 92: 307-328

Pesaran, M. H. and Potter, S. M. (1997) A Floor and Ceiling Model of U. S. Output. Journal of Economic Dynamics and Control 21: 661-695

${ }^{18}$ See Escribano and Jorda (1999) and Teräsvirta(1998). 
Ramsey, J. B. (1969) Tests for Specification Errors in Classical Linear Least-Squares Regression Analysis. Journal of the Royal Statistical Society (B) 31: 350-371

Rothman, P. (1991) Further Evidence on the Asymmetric Behavior of Unemployment Rates over the Business Cycle. Journal of Macroeconomics 13: 291-298

Saikkonen, P., Luukkonen, R. (1988) Lagrange Multiplier Tests for Testing Nonlinearities in Time Series Models. Scandinavian Journal of Statistics 15: 55-68

Teräsvirta T. (1998) Modelling Economic Relationships with Smooth Transition Regressions. In: Ullah A. and Giles, D.E.A. (eds.) Handbook of Applied Economic Statistics. Marcel Dekker, New York

Teräsvirta, T. (1994) Specification, Estimation and Evaluation of Smooth Transition Autoregressive Models. Journal of the American Statistical Association 89: 208-218

Teräsvirta, T., Anderson, H. M. (1992) Characterizing Nonlinearities in Business Cycles Using Smooth Transition Autoregressive Models. Journal of Applied Econometrics 7: S119-S136

Teräsvirta, T., Tjøstheim, D., Granger, C. W. J. (1994) Aspects of Modelling Nonlinear Time Series. In: Engle, R. F., McFadden, D. (eds.) Handbook of Econometrics, Vol. 4. Elsevier, Amsterdam

Tong, H. (1983) Threshold Models in Non-Linear Time Series Analysis. Springer, Berlin Heidelberg New York

Tsay, R. (1989) Testing and Modelling Threshold Autoregressive Processes. Journal of the American Statistical Association 84: 231-240

Van Dijk, D., Franses, P. H., Lucas, A. (1998) Testing for Smooth Transition Nonlinearity in the Presence of Additive Outliers. Journal of Business and Economic Statistics 17(2): 217-235

White, H. (1984) Asymptotic Theory for Econometricians. Academic Press, London and San Diego 